\title{
A NOTE ON NOETHERIAN P.I. RINGS
}

\begin{abstract}
AMIRAM BRAUN
AbStRact. Let $R=F\left\{x_{1}, \ldots, x_{k}\right\}$ be an affine semiprime p.i. ring, $\operatorname{Krull}-\operatorname{dim}(R)$ $=2$ and $F$ a central subfield. It is proved that $R$ is noetherian iff $R$ is a left (right) finite module over a commutative affine subring.
\end{abstract}

Introduction. In [C] and later in [Sch] examples were given (for the first time) of prime affine Noetherian p.i. rings which are not finite modules over their centers. The common feature that both examples share is that they are finite modules over a commutative Noetherian subring. Also, both examples are of Krull dimension 2. We show here that this is not accidental. Indeed we have the following

THEOREM. Let $R=F\left\{x_{1}, \ldots, x_{k}\right\}$ be a semiprime affine p.i. ring, $k \cdot d(R)=2$. Then $R$ is noetherian iff $R$ is a finite left (right) module over a commutative affine (hence noetherian) subring.

For notations we follow [P]. Also by $N_{R}(I)$ we denote the nilradical of the ideal $I$ of $R . T(R)$ will denote the "trace envelope" of $R($ e.g., $[\mathrm{B}]), k \cdot d(R)$ will denote the classical Krull dimension of $R$, and $h_{R}(I)$ will denote the height of the ideal $I$ in $R$.

LEMMA 1. Let $R=F\left\{x_{1}, \ldots, x_{k}\right\}$ be a semiprime affine p.i. ring with $k \cdot d(R)=$ 1. Then $R$ is a finite module over its center.

Proof. Let $P_{1}, \ldots, P_{l}$ be the minimal prime ideals in $R$; we have $\cap_{i=1}^{\prime} P_{i}=$ $\{0\}$. Let $R_{i} \equiv R / P_{i}$ for $i=1, \ldots, l$. Then $R_{i}$ is a finite module over its center $Z_{i}$ [S, Sch]. We have that $\bigcap_{j \neq i} P_{j}+P_{i} / P_{i} \equiv \overline{\bigcap_{j \neq i} P_{j}} \neq 0$ in $R_{i}$ for $i=1, \ldots, l$. By Noether's normalization theorem, [N] there exists $\bar{a}_{i} \in \overline{\bigcap_{j \neq i} P_{j}} \cap Z_{i}$ such that $Z_{i}$ is integral over $F\left[\bar{a}_{i}\right]$ for $i=1, \ldots, l$ (if $k \cdot d\left(R_{t}\right)=0$ we take $\bar{a}_{t}=1$ ). Let $\nu: R \rightarrow R_{1}$ $\oplus R_{2} \oplus \cdots \oplus R_{l}$ denote the obvious inclusion with $\nu(x)=\bar{x}_{1} \oplus \cdots \oplus \bar{x}_{l}$ where $\bar{x}_{i}$ denotes the canonical image of $x$ in $R_{i}$. Let $a_{i} \in \bigcap_{j \neq i} P_{j}$ be a preimage of $\bar{a}_{i}$ in $R, \quad i=1, \ldots, l$; then $\nu\left(a_{i}\right)=\bar{a}_{i}$, and $\nu\left(a_{i}\right) \in Z_{i} \subseteq Z\left(R_{1} \oplus \cdots \oplus R_{l}\right)$. Thus $F\left[\nu\left(a_{1}\right), \ldots, \nu\left(a_{l}\right)\right]$ is a central subring of $R_{1} \oplus \cdots \oplus R_{l}$. Moreover $Z_{1}$ $\oplus \cdots \oplus Z_{l}=Z\left(R_{1} \oplus \cdots \oplus R_{l}\right)$ is integral over $F\left[\nu\left(a_{1}\right), \ldots, \nu\left(a_{l}\right)\right]$ and consequently $\nu(R) \subseteq R_{1} \oplus \cdots \oplus R_{l}$ is a finite module over $\nu\left(F\left[a_{1}, \ldots, a_{l}\right]\right)$. Q.E.D.

Let $R$ be a semiprime affine p.l. ring with minimal prime ideals $P_{1}, \ldots, P_{k}$. Assume that p.i.d $\left(P_{j}\right)=n_{t}$ for $i_{t-1}+1 \leqslant j \leqslant i_{t}, \quad 1 \leqslant t \leqslant l$, and $n=n_{1}>n_{2}$ $>\cdots>n_{l}, i_{l}=k$. Let $Q_{t}=\cap_{j=i_{t-1}+1}^{i_{t}} P_{j}, t=1, \ldots, l$. By [A] there exists a

Received by the editors September 11, 1980.

1980 Mathematics Subject Classification. Primary 16A38; Secondary 16A33.

() 1981 American Mathematical Society 0002-9939/81/0000-0551/\$01.75 
central alternating polynomial $\alpha_{t}$ of $n_{t} \times n_{t}$ matrices with the following properties:

(a) If $\alpha_{t}=\alpha_{t}\left[X_{1}, \ldots, X_{m}, Y_{1}, \ldots, Y_{s}\right]$ then given $\bar{r} \in R / Q_{t}$ we have $\alpha_{l}\left[b_{1}, \ldots, b_{m}, d_{1}, \ldots, d_{s}\right] c_{i}(\bar{r})=\alpha_{t}\left[f_{1}, \ldots, f_{m}, d_{1}, \ldots, d_{s}\right]$ where the $b_{i}$ 's, $d_{i}$ 's and $f_{i}$ 's are in $R / Q_{t}$ and $c_{i}(\bar{r})$ is the $i$ th coefficient of the characteristic polynomial of $\bar{r}$.

(b) If $e=\alpha_{l}\left[b_{1}, \ldots, b_{m}, d_{1}, \ldots, d_{s}\right]$ then $(e \vec{r})^{k}+\gamma_{1}(e \vec{r})^{k-1}+\cdots+\gamma_{k}=0$ where $\gamma_{i}, i=1, \ldots, k$, are evaluations of $\alpha_{t}$ in $R / Q_{t}$.

Lemma 2. Let $J_{t}=\left\{x \in R \mid x\right.$ is an evaluation of $\alpha_{t}$ with elements in $\left.\bigcap_{j<t-1} Q_{j}\right\}$. Then for $x \in J_{t}$ we have

(i) $x \in Z(R)$.

(ii) if $r \in R$ then $(x r)^{m}+\gamma_{1}(x r)^{m-1}+\cdots+\gamma_{m}=0$ where $\gamma_{i} \in J_{t}, i=$ $1, \ldots, m$.

(iii) $J_{t} \subseteq \cap_{j \neq t} Q_{j}$.

(iv) $J_{t} \cdot J_{s}=0$ for $t \neq s, t, s=1, \ldots, l$.

Proof. (i) $[x, R] \subset Q_{t} \cap \cdots \cap Q_{l}$ since $x \equiv 0\left(\bmod Q_{t+1} \cap \cdots \cap Q_{l}\right)\left(\alpha_{t}\right.$ vanishes on $\left.R / Q_{i}, t+1 \leqslant i\right)$ and $x \equiv$ central element $\left(\bmod Q_{t}\right)$. Also $x \in$ $\cap_{j<t-1} Q_{j}$; thus $[x, R] \subset \cap_{j=1}^{l} Q_{j}=\cap_{j=1}^{k} P_{j}=\{0\}$.

(ii) This is a consequence of properties (a) and (b).

(iii) One merely observes that $x \in \cap_{j<t-1} Q_{j}$ by definition and $x \in \cap_{j>t+1} Q_{j}$ since $\alpha_{t}$ vanishes on $R / Q_{t}$ for $j \geqslant t+1$.

Finally, (iv) is a trivial consequence of (iii).

We now prove our main result:

THEOREM. Let $R=F\left\{x_{1}, \ldots, x_{s}\right\}$ be a semiprime affine p.i. ring $R$ and $k \cdot d(R)$ $=2$. Then $R$ is Noetherian iff $R$ is a finite left (right) module over a commutative affine subring $D$.

Proof. We resume the notations of the previous paragraph. We have that $R / Q_{1} \oplus \cdots \oplus R / Q_{l}$ is Noetherian since $R$ is and, by [Sch], $T \equiv T\left(R / Q_{1}\right)$ $\oplus \cdots \oplus T\left(R / Q_{l}\right)$ is integral over the former ring. Let $\nu$ denote the natural inclusion $\nu: R / Q_{1} \oplus \cdots \oplus R / Q_{l} \rightarrow T$. We have by the previous remarks that $\nu\left(\Sigma_{t} J_{t} R\right)$ is a two-sided ideal in $T$ and

$$
N_{T}\left\{\nu\left(\sum_{t} J_{t} R\right)\right\} \cap \nu(R)=N_{\nu(R)}\left\{\nu\left(\sum_{t} J_{t} R\right)\right\} .
$$

Thus

$$
\frac{\nu(R)}{N_{\nu(R)}\left\{\nu\left(\Sigma_{t} J_{t} R\right)\right\}} \subset \frac{T}{N_{T}\left(\nu\left(\Sigma_{t} J_{t} R\right)\right)}
$$

and the inclusion is integral. Clearly, $h_{Z(T)}\left(\nu\left(\Sigma_{t} J_{t}\right)\right) \geqslant 1$. By Noether's normalization theorem we have $x, y$ with $x, y \in Z(T), x \in \Sigma_{t} \nu\left(J_{t}\right) Z(T)$, and $Z(T)$ is integral over $F[x, y]$. Thus, since

$$
k \cdot d\left(\frac{\nu(R)}{N_{\nu(R)}\left\{\nu\left(\Sigma_{t} J_{t} R\right)\right\}}\right)=1,
$$


there exists an $a \in \nu(R)$ with $y^{m}+\gamma_{1} y^{m-1}+\cdots+\gamma_{n} \in N_{\nu(R)}\left\{\nu\left(\Sigma_{t} J_{t} R\right)\right\}, \gamma_{i} \in$ $F[a], i=1, \ldots, m$. Consequently, by (ii) of the previous lemma there are $\beta_{1}, \ldots, \beta_{s}$ in $\nu\left(\Sigma_{t} J_{t}\right)$ such that $y$ is integral over $F\left[\beta_{1}, \ldots, \beta_{s}, a\right]$. Thus $k[x, y]$ is integral over $F\left[x, \beta_{1}, \ldots, \beta_{s}, a\right] \equiv D \subset \nu(R)$ and $D$ is commutative. Consequently $T$ is a right and left module over $D$ and $\nu(R)$ therefore shares the same property. The other direction is definitely trivial. Q.E.D.

\section{REFERENCES}

[A] S. A. Amitsur, Identities and linear dependence, Israel J. Math. 22 (1975), 127-137.

[B] A. Braun, Affine polynomial identity rings and their generalizations, J. Algebra 58 (1979), $481-494$.

[C] G. Cauchon, Anneaux, semipremiers, noetheriens à identités polynomiales, Bull. Soc. Math. France 104 (1976), 99-111.

[N] M. Nagata, Local rings, Interscience, New York, 1962.

[P] C. Procesi, Rings with polynomial identity, Marcel Dekker, New York, 1973.

[S] L. Small, unpublished.

[Sch] W. Schelter, Integral extensions of rings satisfying a polynomial identity, J. Algebra 40 (1976), 245-257.

Department of MATHEmatics, University of Haifa, HaIfa, IsRael 